\title{
ESTUDIO DE CASO: ENTORNO ORGANIZACIONAL DEL TRABAJO REMOTO
}

\author{
Irusta, Juan \\ Ingrassia, Ramiro Carlos
}

\section{Resumen:}

Con base en el Estudio de Casos Múltiples se explora el entorno organizacional durante el ASPyO y DiSPyO. Esta situación sanitaria aceleró la implementación del trabajo remoto. En consecuencia, se busca la clasificación en factores estimulantes, impulsores o retractores de los resultados obtenidos al observar el entorno organizacional, el puesto de trabajo y el entorno familiar.

Palabras claves: Teletrabajo - TIC - Organización - Socio - Tecnología

\begin{abstract}
:
Based on the Multiple Case Study, the organizational environment is explored during ASPyO and DiSPyO. This health situation accelerated the implementation of remote work. Consequently, the classification in stimulating factors, drivers or retractors of the results obtained by observing the organizational environment, the workplace and the family environment is sought.
\end{abstract}

Keywords: Remote Work - ICT - Organization - Socio - Technology

\section{INTRODUCCIÓN}

El surgimiento del coronavirus COVID-19 y las sucesivas medidas de contención epidemiológicas: como el Aislamiento Social, Preventivo y Obligatorio (ASPyO) y luego, el Distanciamiento Social, Preventivo y Obligatorio (DiSyO) provocaron la aceleración de la incorporación de las Nuevas Tecnologías de la Información y Comunicación (NTIC) en las Organizaciones.

En nuestro país, la incorporación de NTIC en las organizaciones, como todo proceso social, ha sido asincrónico. En forma subyacente, la incorporación anterior conlleva a la automatización de tareas, la cual es la problemática central. Si a este proceso, se le adiciona el aceleramiento, la intensidad de los cambios es muy importante. Como resultado de esto, el primer sector afectado es el denominado Capital Humano.

Desde un punto de vista social, este es el punto más sensible de una organización al cual se le modificó, en el actual contexto sanitario, su espacio más íntimo: el hogar. A 
su vez, para la Administración, esto conlleva a una revisión de los modelos de liderazgo e intensifica el rol de agente de cambio de los Licenciados en Administración.

Durante la Fase 1 del ASPyO, la Organización Mundial del Trabajo - sede Buenos Aires - publica un informe de la situación laboral en Argentina. Dentro de las conclusiones, resalta la perspectiva de género.

En estos días, las mujeres asumen una carga de cuidados extraordinaria dentro de su círculo familiar debido al cierre de escuelas y otros espacios de cuidado. Esta circunstancia puede ser incompatible con su trabajo tal y como está organizado actualmente y exige mayores medidas para aliviar la carga de cuidado de las familias. (2020, p. 30)

Luego, en el mismo período, el Centro de Estudios Distributivos, Laborales y Sociales concluye que el $26 \%$ del empleo total tiene la posibilidad de continuar trabajando desde el hogar. Y más aún, del resto de las actividades señala que se caracterizan con el mayor grado de informalidad, así como menor nivel educativo, cualificación y menor nivel educativo.

En el ámbito estatal, el Instituto Nacional de la Administración Pública (2020) publicó una revisión de las principales recomendaciones de estándares internacionales de trabajo remoto para toda la Administración Pública. Además, dicho organismo promueve la investigación de los resultados obtenidos con esta experiencia ad-hoc para el establecimiento de un protocolo ante catástrofes para toda la Administración Pública.

Como resultado de lo expuesto, desde un abordaje cualitativo con en base en el Estudio de Casos Múltiples se explora el entorno organizacional durante el ASPyO y DiSPyO que aceleraron la implementación del trabajo remoto. Para luego, clasificar los resultados en factores estimulantes, impulsores o retractores de esta modalidad de trabajo.

Además, dicho estudio se enmarca en el proyecto de investigación Evaluación del Perfil Tecnológico del Licenciado en Administración. El mismo tiene como objetivo: "Describir el modo en que el perfil profesional de Lic. en Administración y su conocimiento en TIC generan valor en la implementación de estrategias empresariales en empresas rosarinas".

A continuación, se plantea la problemática, la metodología, los resultados y conclusiones. 


\section{PLANTEAMIENTO DEL PROBLEMA}

La incorporación de NTIC se refleja en dos grandes temáticas organizacionales actuales: Comercio Electrónico y Teletrabajo.

Una organización puede visualizarse como dos esferas yuxtapuestas: FrontOffice y BackOffice. Básicamente, el FrontOffice lo conforman todas aquellas actividades organizacionales que tienen contacto con el cliente. En contraposición, el BackOffice son todas aquellas actividades que prestan soporte a las anteriores.

Con el objetivo de exponer la problemática, puede simplificarse que los primeros impactos del Comercio Electrónico se ubican mayormente en el FrontOffice. En otras palabras, las herramientas tecnológicas como página web, carrito de compras y la geolocalización de pedidos pertenecen al FrontOffice, propias del Comercio Electrónico. Como muestra de la evolución anterior al ASPyO, se expone que según la Cámara Argentina de Comercio Electrónico (CACE): "En los primeros 6 meses del año [2019], el eCommerce en la Argentina facturó \$152.619 millones de pesos, lo que representa un incremento del $56 \%$ con respecto al mismo período $2018 . . . "$

El salto cuantitativo que tuvo esta actividad al inicio de la ASPyO es notable, según Sambucetti (2020) presidente de la Cámara Argentina de Comercio Electrónico:

“Estos resultados confirman que el comercio electrónico en Argentina avanzó en las últimas 6 semanas el equivalente a 2 años, tanto en oferta como en demanda. [...] El estudio revela que más de un $36 \%$ de las órdenes de compra de cada tienda pertenecen a nuevos compradores: de esta manera, el eCommerce toma cada vez más protagonismo y tiene un rol fundamental en la economía argentina"

En contraposición, en el BackOffice, esfera en dónde puede situarse, en gran medida, el teletrabajo no ha tenido la misma evolución que el Comercio Electrónico. Con anterioridad al ASPyO, el teletrabajo o trabajo remoto que se había implementado fue para el FrontOffice. A través de telecentros en donde las empresas tercerizan las actividades propias de: Marketing y Ventas, y Servicios (Porter, 1990). El resto de las actividades de soporte continuaban, mayormente, con la modalidad de asistencia física al trabajo. Casos muy puntuales, tenían la modalidad de trabajo remoto, por ejemplo, aquellas ligadas a la energía dado que el teletrabajo surge en la actividad petrolera. También, la industria del desarrollo de software tenía la modalidad de trabajo remoto a tiempo parcial. Sin embargo, este teletrabajo del BackOffice a diferencia del descrito en el FrontOffice, era en el hogar de los trabajadores en vez de un telecentro. 
Debido a la situación descrita, para categorizar desde un punto de vista científico, esta problemática al centrarse en el Capital Humano tiene una visión social que puede abordarse desde un enfoque socio-tecnológico.

Desde una perspectiva cualitativa, se espera aportar una "fotografía" del contexto en la cual se enmarcó este proceso de consolidación de una posible nueva categoría de empleado: el teletrabajador. Por esto, una pregunta iniciadora o rectora del presente estudio es: ¿cómo se implementa el teletrabajo en las organizaciones en estas condiciones sanitarias?

\section{OBJETIVO}

Ante la situación sanitaria actual se persigue la exploración del contexto organizacional en que se implementa el teletrabajo o trabajo remoto. De esta manera, en forma más detallada:

Describir el entorno organizacional y puesto de trabajo del teletrabajador, Sondear el entorno familiar del anterior, Clasificar los resultados obtenidos en factores estimulantes, impulsores o retractores para el teletrabajo.

\section{METODOLOGÍA}

El presente estudio se enmarca en la Investigación Cualitativa y se elige la estrategia metodológica de Estudio de Casos Múltiples. La Unidad de Análisis es el entorno organizacional de los teletrabajadores argentinos durante el ASPyO. Las dimensiones que se observan en el estudio son: entorno organizacional, puesto de trabajo/teletrabajador y entorno familiar. A su vez, cada una de estas se componen de:

1. Entorno Organizacional: Actividad Económica (CIIU 4 dígitos), Edad de la organización, Capital, Tamaño, Sucursal, Sistema Técnico, Ambiente (Estabilidad, Complejidad, Diversidad y Hostilidad);

2. Puesto de trabajo/Teletrabajador: Nivel Educativo, Adaptabilidad Remota, Equipo Computacional, Sistema de Información que le da soporte, Conexión Remota, habilidades requeridas para el puesto, contexto organizacional requerido del puesto de trabajo, situación de la competencia, tipo de actividad, continuación modalidad remota;

3. Entorno Familiar: Tipo familia, Cantidad Habitaciones (sin cocina y baño), Metros cuadrados totales, Habitaciones por miembro familiar. 
En cuanto a la Unidad de Observación, los casos múltiples se limitan a cuatro teletrabajadores de distintas empresas, de las cuáles dos de estos tenían experiencia en trabajo remoto a tiempo parcial o total.

Además, se utilizó como metodología la triangulación en:

1. Fuente de Datos:

a. Personas: Teletrabajadores para conocer su perspectiva, formas de trabajo y entorno familiar,

i. Nivel agregado: dos casos son independientes el uno del otro,

ii. Nivel interactivo: dos casos tienen relación conyugal, siendo el hogar y una oficina enriquece la perspectiva de la investigación,

b. Espacial: se toman en cuatro organizaciones,

i. Servicio de Banca Minorista,

ii. Generación, transporte y distribución de energía,

iii. Servicios de consultores en informática y suministros de programas de informática,

iv. Servicios de la seguridad social obligatoria.

2. Investigador: quienes suscriben el artículo provienen de dos experiencias distintas: la ciencia y la técnica. También, se refleja en la propensión a las distintas investigaciones: cualitativas y cuantitativas respectivamente. Estos puntos de vista complementarios se reflejan mayormente en la construcción de las dimensiones de la unidad de análisis.

3. Teoría: para la interpretación de los resultados se utilizan dos modelos organizacionales: Cadena de Valor de Michael Porter y Configuraciones de Henry Mintzberg. El objetivo de la utilización de la Cadena de Valor es para identificar la Actividad en donde se encuentra el puesto de trabajo y facilite su comprensión. Asimismo, las configuraciones de Mintzberg se utilizan para dilucidar diferencias entre los expuesto por los entrevistados y la organización. En tal sentido, a partir de los factores situacionales se estimó la configuración y, por ende, las hipótesis de trabajo. Luego, con lo expuesto por el entrevistado se confirman o se descartan tales hipótesis. En este último caso, no se pretende invalidar al entrevistado ni al modelo sino el planteamiento de la diferencia que puede enriquecer las conclusiones del trabajo.

4. Metodología:

a. Intra método (Investigación cualitativa): 
i. Entrevistas Semi Estructurada: con la finalidad de recolectar la percepción y situación de los teletrabajadores seleccionados durante la situación sanitaria,

ii. Observación Participante: uno de los suscriptores está vinculado a los casos estudiados.

b. Inter método

i. Revisión de documentos: datos organizacionales,

ii. Base de Datos: La Occupational Information Network (O*Net) es una base de datos que desglosa cada puesto de trabajo en tareas, contexto organizacional, herramientas y habilidades requeridas. Este último trabajo es realizado por los mismos trabajadores que luego de la estandarización, quedan actualizadas. Además, estos mismos más los reclutadores ponderan la importancia de cada elemento descriptivo del puesto de trabajo. Dicha base de datos es referencia a nivel internacional justamente por la participación a nivel global de los trabajadores y reclutadores. También, de las más de 60 tareas que describen cada puesto de trabajo se toman las primeras 10 que proponen los informes en línea (ponderación mayor al $70 \%)$.

\section{RESULTADOS}

\subsection{Entorno Organizacional}

En el Caso no 1, asume una configuración híbrida de Forma de Producto Relacionada. Se destaca el nivel de digitalización de la empresa y la cultura organizacional que amortiguó la situación sanitaria. Por otro lado, las problemáticas planteadas están dentro de las previstas por el modelo dado la reminiscencia de Burocracia Mecánica en esta configuración híbrida. Esto último ocurre por el fuerte control externo (Banco Central República Argentina, Superintendencia de Seguros de la Nación, Comisión Nacional de Valores, entre otros). En este marco, puede entenderse que el acceso al sistema durante el ASPyO sea a través de escritorio remoto. También, dado el Control Externo, se introdujeron nuevos productos regulados como el Ingreso Familiar de Emergencia (IFE) que ocasionó varios inconvenientes subsanables.

En el Caso nำ 2, se configura como híbrido Buro - Adhocracia Profesional, el núcleo operativo se enmarca como personal esencial por lo que continúo con sus tareas en la empresa. El resto del personal tuvo acceso al sistema por usuario y se coordinaron a través de video conferencias en línea. La caída de las operaciones por la situación 
sanitaria se estima del $30 \%$ y se realizó asistencia integral al personal que por viajes de negocios no pudo retornar al país.

En el Caso n 3, la organización se encuadra dentro de la configuración híbrida de Adhocracia Divisional. Hasta el momento, sólo el personal de Operaciones tenía la modalidad de trabajo remoto parcial: 1 o 2 días a la semana. El resto, se concurría a trabajar en las oficinas del cliente, los cuál es una forma de trabajo remoto híbrido. En este tipo de organizaciones, el núcleo operativo se organiza por proyectos de desarrollo y es común la videoconferencia para la coordinación del trabajo. Además, se complementa con un software de seguimiento para la monitorización del trabajo remoto.

En el Caso ํㅜ 4, la organización es una configuración híbrida de Burocracia Mecánica Pública, la diferencia planteada es que ante la situación sanitaria al personal no esencial se coordina a través de la estandarización de resultados. También, el acceso al sistema fue a través de la cuenta de usuario del sistema.

Tabla 1: Descripción de la Dimensión Entorno Organizacional por cada Caso de Estudio

\begin{tabular}{|l|l|l|l|l|}
\hline \multicolumn{1}{|c|}{$\begin{array}{l}\text { Entorno } \\
\text { Organizacional }\end{array}$} & \multicolumn{1}{|c|}{ Caso no 1 } & \multicolumn{1}{|c|}{ Caso no 2 } & \multicolumn{1}{|c|}{ Caso no 3 } & Caso no 4 \\
\hline $\begin{array}{l}\text { Actividad } \\
\text { Económica }\end{array}$ & $\begin{array}{l}\text { Servicios de la } \\
\text { banca } \\
\text { minorista }\end{array}$ & $\begin{array}{l}\text { Generación, } \\
\text { transporte y } \\
\text { distribución de } \\
\text { energía }\end{array}$ & $\begin{array}{l}\text { Servicios de } \\
\text { consultores en } \\
\text { informática y } \\
\text { suministros de } \\
\text { programas de } \\
\text { informática }\end{array}$ & $\begin{array}{l}\text { Servicios de la } \\
\text { seguridad social } \\
\text { obligatoria }\end{array}$ \\
\hline $\begin{array}{l}\text { Edad de la } \\
\text { Organización }\end{array}$ & 100 años & 12 años & 14 años & 49 años \\
\hline Capital & $\begin{array}{l}\text { Privado y } \\
\text { Nacional }\end{array}$ & $\begin{array}{l}\text { Privado y } \\
\text { Extranjero }\end{array}$ & Privado y Nacional & Público \\
\hline Tamaño & $\begin{array}{l}\text { E000 } \\
\text { empleados }\end{array}$ & $\begin{array}{l}\text { 330 a 350 } \\
\text { empleados }\end{array}$ & 14 a 18 empleados & $\begin{array}{l}14600 \text { a 14800 } \\
\text { empleados }\end{array}$ \\
\hline Sucursal & Sí & NS/NC & No Corresponde & Sí \\
\hline $\begin{array}{l}\text { Sistema } \\
\text { Técnico }\end{array}$ & $\begin{array}{l}\text { Producción } \\
\text { Unitaria/Masiva }\end{array}$ & $\begin{array}{l}\text { Producción } \\
\text { Continua }\end{array}$ & $\begin{array}{l}\text { Producción } \\
\text { Unitaria/Masiva }\end{array}$ & $\begin{array}{l}\text { Producción } \\
\text { Masiva }\end{array}$ \\
\hline Estabilidad & Estable & Dinámico & Estable/Dinámico & Estable \\
\hline Complejidad & Simple & Compleja & Simple & Compleja \\
\hline $\begin{array}{l}\text { Diversidad de } \\
\text { Mercado }\end{array}$ & Diversificado & Integrado & Integrado & Diversificado \\
\hline
\end{tabular}




\begin{tabular}{|l|l|l|l|l|}
\hline Hostilidad & Liberal & sin mención & sin mención & sin mención \\
\hline Poder & $\begin{array}{l}\text { Control } \\
\text { Externo }\end{array}$ & Control Externo & Sin Control Externo & Control Externo \\
\hline
\end{tabular}

Fuente: Elaboración Propia

\subsection{Entorno del Trabajo}

Tabla 2: Descripción de la Dimensión Puesto de Trabajo/Teletrabajador para cada Caso de Estudio

\begin{tabular}{|l|l|l|l|l|}
\hline $\begin{array}{l}\text { Puesto de } \\
\text { Trabajo/ } \\
\text { Teletrabajador }\end{array}$ & \multicolumn{1}{|c|}{ Caso no 1 } & \multicolumn{1}{|c|}{ Caso no 2 } & \multicolumn{1}{|c|}{ Caso no 3 } & \multicolumn{1}{c|}{ Caso no 4 } \\
\hline $\begin{array}{l}\text { Nivel } \\
\text { Educativo }\end{array}$ & Universitario & Universitario & Universitario & Universitario \\
\hline $\begin{array}{l}\text { Personal a } \\
\text { cargo }\end{array}$ & sí & no & no & no \\
\hline $\begin{array}{l}\text { Problemática } \\
\text { laboral }\end{array}$ & $\begin{array}{l}\text { Conexión a } \\
\text { Internet }\end{array}$ & $\begin{array}{l}\text { Conexión a } \\
\text { Internet }\end{array}$ & $\begin{array}{l}\text { Conexión a } \\
\text { Internet }\end{array}$ & $\begin{array}{l}\text { Conexión a } \\
\text { Internet }\end{array}$ \\
\hline $\begin{array}{l}\text { Equipo } \\
\text { Computacional }\end{array}$ & $\begin{array}{l}\text { Equipo } \\
\text { Propio/Empresa }\end{array}$ & Equipo Empresa & Equipo Propio & Equipo Propio \\
\hline $\begin{array}{l}\text { Sistema de } \\
\text { Información }\end{array}$ & $\begin{array}{l}\text { Sistema } \\
\text { Integrado }\end{array}$ & $\begin{array}{l}\text { Sistema } \\
\text { Integrado }\end{array}$ & $\begin{array}{l}\text { Entorno de } \\
\text { Desarrollo }\end{array}$ & $\begin{array}{l}\text { Sistema } \\
\text { Integrado }\end{array}$ \\
\hline $\begin{array}{l}\text { Conexión } \\
\text { remota }\end{array}$ & escritorio remoto & $\begin{array}{l}\text { acceso } \\
\text { restringido }\end{array}$ & $\begin{array}{l}\text { acceso } \\
\text { restringido }\end{array}$ & $\begin{array}{l}\text { acceso } \\
\text { restringido }\end{array}$ \\
\hline $\begin{array}{l}\text { Situación de la } \\
\text { competencia }\end{array}$ & $\begin{array}{l}\text { asimetría en el } \\
\text { tamaño de la } \\
\text { competencia }\end{array}$ & sin conocimiento & sin conocimiento & $\begin{array}{l}\text { sin } \\
\text { conocimieno }\end{array}$ \\
\hline $\begin{array}{l}\text { Tipo de } \\
\text { Actividad }\end{array}$ & $\begin{array}{l}\text { Infraestructura } \\
\text { de la empresa }\end{array}$ & $\begin{array}{l}\text { Infraestructura } \\
\text { de la empresa }\end{array}$ & Operaciones \\
\hline $\begin{array}{l}\text { Continuación } \\
\text { modalidad } \\
\text { remota }\end{array}$ & sí & $\begin{array}{l}\text { Infraestructura } \\
\text { de la empresa }\end{array}$ \\
\hline
\end{tabular}

\section{Fuente: Elaboración Propia}

En el caso nำ1, lo observable por parte del entrevistado es la demora en la entrega de equipo por parte de la empresa. luego de cuatro meses, se entregaron los equipos. Esta situación puede comprenderse dentro de las consecuencias organizacionales del Control Externo. 
En cuanto a la proveeduría de los equipos para el trabajo remoto, en este contexto, es la inversa al movimiento Bring Your Own Device (BYOD) "trae tu propio equipo". El mismo se caracteriza por solicitarle a la empresa, por parte de los empleados, la utilización de sus propios teléfonos celulares o laptops. Si bien esto conlleva un aumento en los costos de seguridad informática, la utilización selectiva de estos dispositivos aumenta la productividad de los empleados. Sin embargo, en esta situación inversa e imperativa, satura los equipos hogareños. En caso no 4, si al cónyuge la empresa no le proveía de una computadora portátil su trabajo y la de aquel pasaba al teletrabajador bajo estudio su productividad hubiera descendido bastante.

De acuerdo con la O*Net el puesto se asimiló al "Financial Managers, Branch or Department". De las 10 tareas relevadas, 3 tareas son interpersonales presenciales. En contraste, por la situación sanitaria, se decidió trabajar presencialmente en dos equipos intermitentemente para que en caso de contagio, un equipo sostenga el trabajo diario. Esta solución, en este puesto mitiga el requerimiento interpersonal requerido, más aún, con las reuniones periódicas por videollamada.

En el caso $\mathrm{n}^{\circ}$ 2, en la misma base de datos, se asimilo el puesto a "Risk Management Specialists", de la cuál 1 tarea es interpersonal presencial. De esta manera, los problemas observables sólo se atribuyen al ingreso reciente del teletrabajador en la organización.

En el caso no 3, con el mismo procedimiento, el puesto relevado es "Software Developers, Systems Software", el cual no se requieren tareas interpersonales presenciales.

Y por último, en el caso n 4, se lo identificó con el puesto de "Auditors" el cuál es similar al caso ํㅡ 3 .

En cuanto al contexto organización requerido según la $\mathrm{O}^{*} \mathrm{Net}$, se destaca el requerimiento de una "conversación cara a cara" como totalmente interpersonal. Para el caso $n^{0} 1$ se requiere en un 99\% todos los días, para el caso oㅡ 2 se necesita el $76 \%$ todos los días, para el caso no 3 se estima en un $65 \%$ todos los días, y para el caso $n^{\circ} 4$ se requiere en un $81 \%$ todos los días. En la actualidad, se sustituye por las videollamadas o contacto telefónico. Especialmente, en el caso $n^{\circ} 4$, en donde las denominadas "constelaciones de trabajo" facilitan el desempeño del cargo.

En contraposición a lo anterior, se debe resaltar que en todos los casos la problemática laboral se centró en la conexión a Internet que dada las circunstancias hay cierta tolerancia en las videollamadas. Más aún, cuando toda la familia necesita conectarse por el trabajo o por educación. En estas ocasiones, pudieron sortear mejor los inconvenientes pautando el horario de trabajo con sus jefes. 


\subsection{Entorno Familiar}

En cuanto a esta dimensión, es donde los cambios tuvieron su mayor intensidad. En comparación con el BYOD, el hogar se convirtió en la oficina diaria. Y más aún, con la privación de la movilidad, las dimensiones de la casa y las habitaciones cobraron una gran importancia. Después de la conexión a Internet, el espacio propio para el trabajo remoto es aquello que "colapsaba". Esto fue común, en menor o mayor medida en todos los casos relevados.

Tabla 3: Descripción del Entorno Familiar para cada Caso de Estudio

\begin{tabular}{|l|l|l|l|l|}
\hline Entorno Familiar & \multicolumn{1}{|c|}{ Caso no 1 } & \multicolumn{1}{c|}{ Caso no 2 } & \multicolumn{1}{c|}{ Caso no 3 } & \multicolumn{1}{c|}{ Caso no 4 } \\
\hline Sobrecarga familiar & Crianza Hija/o & Crianza Hija/o & Crianza Hija/o & Crianza Hija/o \\
\hline Tipo Familia & Nuclear & Nuclear & $\begin{array}{l}\text { Hogar } \\
\text { Monoparental }\end{array}$ & Nuclear \\
\hline $\begin{array}{l}\text { Cantidad } \\
\text { Habitaciones }\end{array}$ & 3 & 4 & 4 & 5 \\
\hline $\begin{array}{l}\text { Habitación por } \\
\text { miembro }\end{array}$ & 1,00 & 1,33 & 2,00 & 1,67 \\
\hline $\begin{array}{l}\text { Metros cuadrados } \\
\text { Total }\end{array}$ & 65 & 65 & 150 & 120 \\
\hline
\end{tabular}

Fuente: Elaboración Propia

Luego, la crianza de los hijos cobro importancia al momento de la decisión de cuándo conectarse para trabajar. En los casos no 1 y nํㅡㄹ 2, se tiene otra casa - habitación con mayor espacio y en cercanía de la familia ampliada, que en el ASPyO no se pudo usufructuar.

De esta manera, la planificación familiar tuvo una ponderación superlativa asociada al trabajo remoto.

\section{CONCLUSIONES Y DEBATE}

En el transcurso del relevamiento de los datos de las Dimensiones de la Unidad de Análisis, se fue construyendo un conjunto de factores que pueden ser estimulantes, impulsores 0 retractores (no controlables por las organizaciones y/o los teletrabajadores).

En base los casos relevados, la variable que no controlan los teletrabajadores ni las organizaciones, factor retractor, es la Prestación de Servicios de Internet. Más aún, con las medidas sanitarias en donde no se tiene mucha margen de planificación laboral.

Luego, se observa que el intempestivo surgimiento de la Pandemia y las medidas sanitarias acrecentaron para el trabajo remoto, aquellos problemas derivados de la 
Planificación familiar y su hábitat. En este análisis, dicha variable es controlable por parte de los teletrabajadores en tanto no estén privados de la circulación. En otras palabras, el trabajo remoto con libre circulación puede ser un factor estimulante.

Si bien, la seguridad informática es un costo que se acrecienta con el trabajo remoto, es una variable controlable o estimulante para las organizaciones. El ASPyO/DiSPyO se pueden tomar como pruebas piloto para replantear las Estrategias de Seguridad Informática. De hecho, tres de los cuatro casos bajo estudio tiene un Control Externo que les obliga a tener estándares de Seguridad.

Para concluir, como factor impulsor es el diseño mismo de los puestos de trabajo y su nivel de digitalización. En tres de los casos estudiados, es posible continuar con este modalidad ampliamente.

\section{REFERENCIAS BIBLIOGRÁFICAS:}

Alexander, A., De Smet, A. y Mysore, M. (7 de julio de 2020) Reimagining the postpandemic workforce. McKinsey\&Company. Recuperado de: https://www.mckinsey.com/business-functions/organization/ourinsights/reimagining-the-postpandemic-workforce\#

Cámara Argentina de Comercio Electrónico (26 de mayo de 2020) El comercio electrónico creció un 84\% en abril. SL: CACE. Recuperado de: https://www.cace.org.ar/noticias-el-comercio-electronico-crecio-84-en-abril

Cámara Argentina de Comercio Electrónico (28 de agosto de 2019) Crece el eCommerce en el primer semestre del año. SL: CACE. Recuperado de: https://www.cace.org.ar/noticias-crece-el-ecommerce-en-el-primer-semestre$\underline{\text { del-ano }}$

Centro de Estudios Distribuidos, Laborales y Sociales [CEDLAS] (2020). El Impacto Asimétrico de la Cuarentena (Documento de Trabajo ํㅜ261). La Plata: Recuperado de https://www.cedlas.econo.unlp.edu.ar/wp/wpcontent/uploads/doc cedlas261.pdf?dl=0

Ernst, C. y López Mourelo, E. (2020) La COVID-19 y el mundo del trabajo en Argentina: impacto y respuestas de política. CABA: Recuperado de https://www.ilo.org/wcmsp5/groups/public/---americas/---ro-lima/---ilobuenos aires/documents/publication/wcms 740742.pdf

Facultad Latinoamericana de Ciencias Sociales [FLACSO] - Sede Argentina (2020) Un Balance Preliminar de la Crisis Económica en la Argentina en el Marco del Coronavirus (Documento de Trabajo no 17). SL: Recuperado de 
https://www.flacso.org.ar/wp-content/uploads/2020/06/Crisis-coronavirus DT-

FLACSO AEYT-CIFRA junio2020.pdf

Instituto Nacional de la Administración Pública [INAP] (2020). Trabajo remoto: hacer de necesidad virtual (Informe oㅜ 30). CABA: Recuperado de

https://www.argentina.gob.ar/noticias/cuinap-30-trabajo-remoto-hacer-denecesidad-virtud-oscar-oszlak

Mintzberg, H. (1991). Diseño de Organizaciones Eficientes (2da reimpresión). Buenos Aires: EI ATENEO.

Pendersen, C. y Ritter, T. (10 de Abril de 2020) Preparing your business for a postpandemic world. Harvard Busisnes Review. Recuperado de:

https://hbr.org/2020/04/preparing-your-business-for-a-post-pandemic-world

Porter, M. (1991). Ventaja Competitiva: creación y sostenimiento de un desempeño superior. Buenos Aires: Editorial Rei Argentina S.A.

Rubbini, N. (2018). Organizaciones que implementan teletrabajo: recomendaciones para facilitar las relaciones sociales satisfactorias en el trabajo (Tesis Doctoral). Universidad Nacional de La Plata: La Plata.

U.S. Department of Labor, Employment \& Training Administration (18 de agosto, 2020). Occupational Information Network [Base de Datos en linea]. Recuperado de: https://www.onetonline.org/ 